\title{
As representações sociais da infecção hospitalar elaboradas por profissionais de enfermagem
}

\author{
The social representations of hospital-aceuired infections elaborated by nursing professionals
}

Las representaciones sociales de la infección hospitalária elaboradas por profesionales de enfermería

\section{Ana Maria Ribeiro dos Santos', Luciane dos Anjos Formiga Cabral', David Soares Brito', Maria Zélia de Araújo Madeiral", Maria Enoia Dantas da Costa e Silva', Maria do Carmo de Carvalho e Martins ${ }^{1}$ \\ 'Faculdade NOVAFAPI, Teresina, PI \\ "Universidade Federal do Piauí. Teresina, PI}

Submissão: 19/03/2008

Aprovação: 3 1/07/2008

\section{RESUMO}

O controle e a prevenção das infecções hospitalares constituem-se um constante desafio para os profissionais de enfermagem. Este estudo objetivou apreender as representações sociais das infecções hospitalares elaboradas a partir da percepção dos profissionais de enfermagem e discutir como essas representações influenciam as suas práticas. Trata-se de uma pesQuisa Qualitativa, realizada em um serviço de urgência e emergência de referência, utilizando a entrevista semi-estruturada para coleta dos dados com posterior análise categorial temática. Observou-se Que a execução das atividades está intimamente relacionada com as subjetividades individuais de cada profissional e profundamente dependente dos seus valores morais, éticos, ideológicos e subjetivos, envolvendo interpretação, ajuizamento e decisão pessoal na aplicação do conhecimento científico.

Descritores: Infecção Hospitalar; Prevenção e controle; Enfermagem.

\begin{abstract}
Prevention and control of hospital aceuired infections is a constant challenge for the nursing professionals. This study sought to grasp the social representations of hospital-aceuired infections elaborated from the perceptions of nursing professionals, and to discuss how these representations influence their practices in controlling and preventing infections. This is a Qualitative research, applied in a reference urgency-emergence service, which used as a data collection technique the semi-structured interview, and for content analysis the category elaboration. It was observed that the execution of the activities is closely tied to the individual subjectivities of each professional and deeply dependant on the moral, ethical, ideological and subjective values of these professionals, regarding interpretation, judging and personal decision in the application of the scientific knowledge.
\end{abstract}

Descriptors: Hospital Infection; Prevention and Control; Nursing.

\section{RESUMEN}

El control y la prevención de las infecciones hospitalares se constituyen en un desafío constante para los profesionales de enfermería. Esto estudio objetivó aprehender las representaciones sociales de las infecciones hospitalares elaboradas a partir de la percepción de los profesionales de enfermería, y discutir como esas representaciones influencian en sus prácticas. Se trata de una pesQuisa cualitativa, realizada en un servicio de urgencia y emergencia de referencia, utilizando la entrevisa semi-estruturada para colecta de los datos con posterior análisis categorial temático. Fue observado Que la ejecución de las actividades está intimamente relacionada con las subjetividades individuales de cada profesional y profundamente dependiente de sus valores morales, éticos, ideológicos y subjetivos, envolviendo interpretación, ajuizamento y decición personal en la aplicación del conocimiento científico.

Descriptores: Infecicón Hospitalar; Prevención y control; Enfermería. 


\section{CONSIDERAÇÕES INICIAIS}

Com o desenvolvimento da biologia, da microbiologia e a conseqüente descoberta dos microorganismos causadores de doenças, a área da saúde, em especial a medicina especializada, teve grande impulso, principalmente nos aspectos biológicos e tecnológicos do conhecimento. Desde então, muito se tem feito: estudos, pesquisas, cursos, drogas, materiais e equipamentos de proteção individual, entre outros, como medidas de prevenção e controle de Infecção Hospitalar.

No Brasil, ao longo dos anos, a IH constituiu-se um problema de Saúde Pública, necessitando de intervenção do governo, através do Ministério da Saúde, instituindo medidas especificas. Assim instituiu-se políticas de saúde para a área hospitalar como a criação de Comissões de Controle de Infecções Hospitalares (CCIH) e ações educativas como treinamentos e cursos específicos, centrados nos aspectos técnicos e biológicos, voltados para os profissionais de saúde.

Os hospitais, além de equipamentos adequados, adotam medidas habituais de prevenção e controle de infecções, mas devem lembrar Que toda a comunidade hospitalar é um agente importante e determinante neste controle. Ressaltamos aQui o profissional de enfermagem por ser o principal agente do cuidado direto com o cliente.

Apesar de todos os esforços, do desenvolvimento e de investimentos dos vários segmentos: laboratórios, empresas e indústrias de tecnologias, instituições hospitalares, profissionais de saúde e órgãos governamentais, no controle e prevenção da $\mathrm{IH}$, observa-se Que os seus índices persistem elevados, em uma realidade pouco modificada, e ainda com o surgimento de novos problemas, tais como: multirresistência de microorganismos, seleção de flora hospitalar, antimicrobianos Que não são mais utilizados, por não mais atuarem, entre outros. Constituindo-se em novos desafios no controle de infecções para todos os profissionais da saúde. Dentre estes problemas ressaltamos o surgimento das bactérias multirresistentes como agravante das infecções hospitalares, tendo como causa principal o uso indiscriminado e indevido de antibióticoterapia.

Hoje, com todo o conhecimento/tecnologia disponível, incluindo várias medidas profiláticas comprovadamente eficazes, permanece ainda para nós, o desafio de Semmelweis, de tornar as ações de prevenção e controle das infecções práticas rotineiras nas instituições de saúde. É primordial entender porQue as medidas de prevenção e controle adotadas (sejam recursos materiais ou práticas educativas) não repercutem na mudança dos índices de IH nos serviços de saúde.

No entanto, a educação como a principal forma de divulgação e disseminação de conhecimento e informações não tem conseguido modificar comportamentos e condutas específicas; sabemos Que as medidas de prevenção e controle de infecções adotadas não repercutem na mudança dos índices de infecção hospitalar. Esperase efetivamente Que as ações educativas possibilitem ou estimulem a reflexão da atuação de cada um, propicie a aprendizagem e modifieue as práticas instituídas.

Dada à importância da repercussão das $\mathrm{IH}$ no contexto hospitalar e social, é premente a necessidade de se conhecer as representações sociais do grupo em estudo Que possam estar contribuindo para manutenção da realidade atual e dessa forma encontrar meios para adoção de medidas Que interfiram e solucionem este problema.

Por isso buscamos trabalhar com as representações sociais como alternativa para entender Qual a contribuição do profissional de enfermagem na manutenção dos índices de infecção hospitalar através das representações sociais destes profissionais sobre infecção hospitalar. E, ainda, Qual a interferência das representações sociais no trabalho como medida de prevenção e controle de infecção nos serviços de saúde. Considerando todos estes aspectos, definimos como objeto de estudo apreender as representações sociais da infecção hospitalar, elaboradas por profissionais de enfermagem do Serviço de Urgência e Emergência.

Através desta pesQuisa objetivou-se apreender as representações sociais elaboradas por profissionais de enfermagem sobre infecção hospitalar; identificar os determinantes da não adesão dos profissionais de enfermagem às técnicas e rotinas instituídas para controle e prevenção das infecções hospitalares a partir das representações sociais da IH e discutir como essas representações influenciam as suas práticas no controle e prevenção das infecções, para Que possam nortear programas efetivos de elaboração das práticas educativas nos serviços de saúde.

\section{BASES TEÓRICO-METODOLÓGICAS}

\section{Contextualizando as Representações Sociais}

Nos últimos anos o conceito de representações sociais tem aparecido com freeüência em trabalhos de diversas áreas, e isto vem despertando o interesse dos profissionais de saúde pelo tema, buscando entendê-lo.

Representar pode dizer muitas coisas de várias formas dependendo do contexto inserido e considerando que as sociedades, comunidades são constituídas por pessoas, e estas são seres humanos que possuem ideais, desejos, escolhas, ambições, além de viverem ou sofrerem influências de fatores como: ambiente, trabalho, família.

A preocupação com as representações sociais nos estudos relacionados à área da saúde é recente, uma vez Que estas originaram-se no campo da psicologia e sociologia; enquanto a saúde foi sempre muito direcionada para técnicas, métodos, fórmulas e diagnósticos. Na enfermagem vários autores têm enfatizado a importância de se observar o sujeito em sua totalidade de processos intelectuais, emocionais, afetivos e culturais para tornar possível maior efetividade nas intervenções e mudanças de condutas.

A representação social é um conjunto de conceitos, explicações e afirmações Que se originam no cotidiano através de comunicações inter-individuais, Que contribui para a formação de condutas e a orientação das comunicações sociais ${ }^{(1)}$. Desde a origem da formulação proposta por Moscovici, em 1961, as representações sociais são caracterizadas como fenômenos complexos Que diz respeito ao processo pelo Qual o sentido de um dado objeto é estruturado pelo sujeito, no contexto de suas relações ${ }^{(2)}$.

O saber popular é a base para construção do conhecimento em Qualquer área, uma vez Que cada indivíduo, grupo, comunidade possui uma definição/conceito pré-estabelecida acerca de um objeto. Assim, "o Que Moscovici avança, com esta sistematização, é uma reabilitação do senso comum, do saber popular, do conhecimento do cotidiano, o conhecimento "pré-teórico" de que falam Berger e Luckmann"(3). 
Neste processo, informações de diferentes ordens são continuamente elaboradas, transformadas, recriadas, articulando instâncias, níveis e dimensões numa síntese Que permite ao sujeito agir e interagir, situar-se e se definir, negociar aceitação estabelecendo proximidades e diferenças ${ }^{(4)}$.

A elaboração e funcionamento de uma representação podem ser compreendidos através dos processos de objetivação e ancoragem Que compreendem a imbricação e articulação entre atividade cognitiva e as condições sociais em Que são elaboradas as representações. Fundamentalmente, a objetivação consiste em materializar as abstrações, corporificar os pensamentos, tornar físico e visível o impalpável, enfim, transformar em objeto o Que é representado. lá a ancoragem permite a incorporação do Que é desconhecido ou novo em uma rede de categorias usuais ${ }^{(2)}$.

Em relação aos conceitos psicológicos, existe uma tendência a se utilizar diferentes noções, tais como atitude, opinião, imagem, de modo indiferenciado, e, até mesmo, como noções sinonímicas de representações. É necessário observar as distintas abordagens, pois essas diferenças determinam, ao mesmo tempo, o conteúdo da observação e as conseQüências teóricas Que delas são deduzidas $^{(2)}$.

A representação social deve ser estudada articulando elementos afetivos, mentais e sociais, e integrando, ao lado da cognição, da linguagem e da comunicação, as relações sociais Que afetam as representações e a realidade material, social e ideal (das idéias) sobre a Qual elas vão intervir ${ }^{(4)}$.

Nesta construção o saber vai sendo interiorizado e organizado, constituindo-se em um sistema dominante de pensamentos e relações Que se incorporam ao saber dos grupos sociais aos quais os indivíduos pertencem. Assim, toda representação social é a representação de alguma coisa, algo ou alguém e tem a função de transformar algo não familiar, ou a própria não-familiaridade, em familiar ${ }^{(5)}$.

No contexto da prevenção e controle das infecções hospitalares a utilização do embasamento teórico das representações sociais, é fundamental e indispensável para compreensão das subjetividades envolvidas, uma vez Que as representações elaboradas por indivíduos/profissionais determinam ao mesmo tempo suas condutas e seus comportamentos, contribuindo para manutenção dos índices de infecção hospitalar.

\section{METODOLOGIA}

Trata-se de uma pesquisa Qualitativa, Que busca a partir de uma realidade construir um saber compreensivo e interpretativo Que capte os significados, valores, crenças, sentimentos e atitudes, o Que corresponde a um espaço mais profundo das relações, dos processos e dos fenômenos Que não podem ser reduzidos à operacionalização de variáveis( ${ }^{(6)}$.

Os sujeitos participantes foram auxiliares e técnicos de enfermagem escolhidos aleatoriamente na equipe de enfermagem Que trabalha no Serviço de Pronto Socorro do Hospital Getúlio Vargas (HGV), uma instituição pública, hospital geral de grande porte, situado em Teresina-Piauí. O HGV possui na sua estrutura o Serviço de Urgência e Emergência, Que se constitui um serviço de referência estadual e regional, pela oferta de várias especialidades médicas, da alta complexidade e de seu poder resolutivo. Este, portanto, foi o local onde a pesquisa se desenvolveu.
A técnica para coleta dos dados foi à entrevista semi-estruturada. As entrevistas foram gravadas em fita cassete, posteriormente transcritas e analisadas utilizando-se a análise categorial temática. A participação de cada sujeito neste estudo contou com o respaldo legal do consentimento livre e esclarecido, conforme a Resolução 196/96 Que trata da ética de pesquisa com seres humanos ${ }^{(7)}$.

\section{RESULTADOS E DISCUSSÃO}

O objeto de estudo foi representado pelos sujeitos da pesQuisa através das seguintes categorias: conhecimento sobre infecção hospitalar; condições de trabalho relacionadas ao controle de infecção hospitalar e sentimentos dos profissionais.

No processo de categorização dos dados foram definidas as categorias com o objetivo de fornecer uma representação simplificada dos dados, pontuando as relações entre elementos cognitivos, práticas e investimentos afetivos.

\section{Conhecimento sobre Infecção Hospitalar}

O conteúdo das falas agrupadas semanticamente nesta categoria com sete (07) unidades de registro, evidencia o conhecimento dos profissionais de enfermagem (técnicos / auxiliares) sobre infecção hospitalar, remetendo à formulação de um conceito sobre infecção hospitalar.

Resumidamente, conceituamos infecção hospitalar como Qualquer processo infeccioso adeuirido no ambiente hospitalar. A Portaria MS 2.616/98 Que regulamenta as ações de controle de infecção hospitalar no país, esta é definida como infecção adQuirida após admissão do paciente no hospital e Que se manifesta durante a internação ou após a alta, desde Que sejam relacionadas com a internação ou procedimentos realizados no hospital|(8).

No presente trabalho, a maioria dos profissionais de enfermagem representa a infecção hospitalar como contaminação adQuirida no hospital, através dos materiais e eQuipamentos. A valorização dos materiais permanentes está relacionada com a macroscopia da sujidade, porQue são visíveis a olho nu, ao contrário dos germes causadores da infecção hospitalar. Em algumas falas a IH foi atribuída à contaminação por germes e falta de limpeza.

...ocorrem através de contaminações dos germes através de materiais contaminados... (E-1).

\section{É uma forma de contaminação Que o paciente adquire no} hospital. (E-1 I)

Realmente, o aspecto de limpeza impressiona, causa bem estar físico e psicológico aos profissionais e pacientes. Por outro lado o aspecto de limpo pode induzir os profissionais a pensarem Que aquele local ou objeto está livre de germes, reduzindo a preocupação, o cuidado e a vigilância com outros meios de contaminação, relaxando nas medidas normais de prevenção e controle das IH. Pois, na limpeza os microorganismos não são destruídos. Os equipamentos devem estar limpos antes da desinfecção ou esterilização(9).

Ficou evidenciada na maioria das falas eue os profissionais de enfermagem atribuem à causa da infecção hospitalar à contaminação através de recursos materiais. Associam, Que se tem infecção é 
porQue houve contaminação de alguma forma. Como esta contaminação acontece é verbalizado na referência freqüente a utensílios como: colchões, lençóis e equipamentos.

Desinfecções de camas, mesa de cabeceiras, pois temos pacientes com HIV, meningite, varicela, neste caso Quando ele sair deve ser feita uma desinfecção bem feita para ser usado o leito. (E-1)

Assim os profissionais de enfermagem objetivam as representações sociais da infecção hospitalar pela palavra contaminação. A interpretação de infecção hospitalar, analisada sob a ótica das representações sociais é uma reprodução da vivência destes funcionários com um significado particular, elaborada a partir de suas opiniões e percepções. As opiniões são estreitamente ligadas à pessoa, extremamente diversas e facilmente submetidas à influência das situações encontradas - daí sua capacidade de mudança ${ }^{(10)}$.

Temos assim alguns fundamentos na construção deste saber prático - definição de infecção hospitalar, considerando o processo de formação das representações sociais. As representações são um conjunto de conceitos, afirmações e explicações Que se originam na vida diária durante os processos comunicacionais interpessoais obtidos nas relações cotidianas ${ }^{(5)}$.

Percebeu-se ainda Que todos associam a representação de infecção hospitalar ao hospital (instituição), estando esta relacionada ou não aos procedimentos realizados. Tal conhecimento é préestabelecido, ressaltado pelos trechos das falas dos sujeitos e evidenciado como demonstrado abaixo:

\section{É a infecção adQuirida no próprio hospital. (E-7) \\ É a infecção adQuirida no hospital. (E-9) \\ É aquela que o paciente adquire no hospital. (E-10)}

\section{Condições de Trabalho Relacionadas ao Controle de Infecção Hospitalar}

Nesta categoria foram destacadas sete (7) falas, onde as representações sociais dos sujeitos sobre as condições de trabalho são objetivadas pela interferência da estrutura organizacional e funcional nas atividades de enfermagem. Estão ancoradas em conhecimentos elaborados a partir de experiências, vivências desses profissionais, os Quais orientam suas condutas, procedimentos frente às medidas de controle e prevenção da infecção hospitalar, contribuindo para realidade atual dos índices de IH.

Em países como o Brasil, onde há escassez de recursos destinados à saúde, o controle da infecção hospitalar, além de atender às exigências legais e éticas, é também um problema socioeconômico, pois se tem investido em tecnologia cara, tanto na pesquisa (equipamentos, microbiologia, etc.) como na produção de novas drogas antimicrobianas. A IH aumenta o tempo de internação do paciente, encarecendo assim o custo da hospitalização pelo uso dos recursos hospitalares e de antimicrobianos, além do risco imposto ao paciente. $\mathrm{O}$ funcionário reconhece isto Quando afirma:

$$
\text { É silenciosa e prejudicial para o paciente. (E-2) }
$$

\section{É prejudicial para o paciente e para o funcionário. (E-3)}

A unidade de emergência de instituições com economia limitada dificilmente possuem uma planta física Que permita separar pacientes. Essa característica aumenta a probabilidade de transmissão de microorganismos por meio de contato ou vias aéreas $^{(1)}$. Além do Que, isto interfere na prática profissional e contribui para exposição ocupacional dos profissionais de saúde, impondo riscos ao ambiente de trabalho; nos fragmentos dos discursos dos sujeitos ficou evidenciado o posicionamento dos profissionais diante das dificuldades impostas, como observado nesta fala:

Às vezes chega paciente em parada e não dá tempo a gente se equipar rapidamente, a gente faz o Que é necessário embora muitas vezes a gente sabendo que é errado. $(\mathrm{E}-5)$

Talvez um dos fatores mais importantes relacionados aos cuidados de urgência é que na maioria das vezes, não se sabe se o paciente é ou não portador de doença transmissível. Os dois pontos principais em atendimento de urgência para prevenção de exposição ocupacional desnecessária são: implementação de precauçõespadrão e reconhecimento de sinais e sintomas para implementação de medidas preventivas especificas ${ }^{(11)}$.

A qualidade da estrutura física existente para a prestação dos cuidados de enfermagem constitui em importante condição para a prevenção e controle da infecção hospitalar, bem como as condições dos recursos materiais utilizados Que estarão em contato com o paciente.

Neste trabalho ressalta-se a preocupação dos profissionais de saúde com os materiais permanentes, como causa determinante das infecções hospitalares. Estes atribuem a esses veículos maior importância em detrimento de outros meios de contaminação, conforme a seguinte fala:

Pra mim a infecção hospitalar é a falta de equipamentos para o trabalho...se houvesse equipamentos adequados para que isso fosse realizado, mais colchões e colocasse melhor os materiais...para a gente poder trabalhar com tranQüilidade para Que não houvesse tanta infecção. (E-5)

O ambiente ou objeto no Qual um micróbio pode sobreviver e em alguns casos multiplicar-se constitui um dos elos da cadeia de transmissão da infecção, Que é o reservatório ${ }^{(9)}$. Existem inúmeros reservatórios contagiosos no ambiente em Que se prestam serviços de saúde, entre eles Quaisquer pacientes, visitantes e membros da equipe, ou móveis, equipamentos, fármacos, água, alimentos e sangue. Os funcionários de enfermagem possuem o conhecimento da importância do controle destes reservatórios e expressam seus posicionamentos para o controle da infecção hospitalar da seguinte forma:

...Que os acompanhantes não deite junto com os pacientes, isto ajudaria muito. (E-4)

Tem Que controlar o grande acesso de pessoas junto aos pacientes. (E-7) 
Ficou evidenciado Que os sujeitos se posicionam a respeito da influência da estrutura organizacional e funcional da instituição hospitalar, Que esta interfere diretamente nas ações dos profissionais de enfermagem e, portanto na assistência ao paciente, seja por falta/insuficiência de materiais ou desobediência às normas e rotinas do serviço. De QualQuer modo expõe os profissionais e pacientes a riscos evitáveis, Que são percebidos e representados pelos próprios profissionais de enfermagem como problemas inerentes ao serviço.

A discussão dos dados obtidos aponta a necessidade de uma definição mais clara acerca das medidas de controle de infecção hospitalar e de um sistema de informação atualizado, acessível às instituições de saúde e seus funcionários. Os dados indicam a importância de programas permanentes de treinamento, necessidade de maiores esclarecimentos sobre a participação de cada indivíduo no controle de infecção hospitalar, para Que, efetivamente, todos os aspectos técnicos conhecidos como indicadores de Qualidade sejam adotados ${ }^{(12)}$.

\section{Sentimentos dos Profissionais}

Esta categoria foi marcante, pois está presente na maioria das falas dos sujeitos a representação social da infecção hospitalar objetivada pela necessidade de conscientização dos profissionais.

É constante a utilização da palavra "conscientização" Quando trabalhamos aspectos subjetivos do ser humano, neste caso profissionais de enfermagem, por Que existe uma necessidade de Que além do conhecimento técnico, ele valorize, aceite os conceitos como verdades e os incorporem em sua prática cotidiana. A palavra aparece em várias falas dos sujeitos, constituindo uma representação da necessidade que os profissionais sentem de mudar conceitos e condutas.

Considerando que ao tomar consciência o homem conhece e julga sua própria realidade, então ele estabelecerá sua forma de agir diante das situações, conforme seus valores e convicções incorporados. Isto determina o comportamento de cada um, como citado:

...acho mesmo é comodismo da parte deles que sabem que estão se contaminando e levando contaminação para suas casas e seus familiares. (E-I)

O uso de EPI's é uma Questão de consciência, um hábito Que deve ser cultivado para proteção do profissional, do paciente e da família (do funcionário)... (E-8)

Nos últimos anos, mais especificamente após o surgimento da AIDS, a literatura especializada em controle de infecção vem abordando, incansavelmente, a necessidade de conscientização dos profissionais da área da saúde, o Que resultaria em mudança de comportamento para uma prática efetiva de medidas preventivas. Essa mudança no comportamento coletivo não tem correspondido às expectativas, apesar da intensa conclamação e do conhecimento disponível. E não faltam razões, Que vão da inexistência de recursos materiais a não percepção do risco ${ }^{(13)}$.

A adoção de novos comportamentos, como a mudança de comportamentos antigos e prevenção de comportamentos indesejáveis, está relacionada à percepção do risco e do benefício
Que isto trará. No entanto, mesmo assim, existem os profissionais de saúde Que banalizam as medidas de prevenção e controle de infecção hospitalar, como por exemplo, a lavagem das mãos. Procedimento este, Que é reconhecidamente um dos caminhos para redução da incidência das infecções hospitalares ${ }^{(14)}$.

Nesse sentido, estudo realizado em uma Unidade de Terapia Intensiva de um hospital de ensino evidenciou Que 18,24\% de infecções poderiam ser preveníveis se o Programa de Controle de IH fosse bem desenvolvido e as precauções fossem adotadas. Desta forma, se evitaria as infecções cruzadas, transmitidas, provavelmente, pelas mãos dos profissionais da saúde, por não usarem a técnica correta da lavagem das mãos, caracterizada, isoladamente, como a ação mais importante para a prevenção e controle das $\mathrm{IH}^{(15)}$.

Este tipo de comportamento é justificado nas falas:

....eu acho que o principal motivo da infecção é justamente a conscientização... a Questão da consciência...e digamos que conscientizar para ver o Que acontece por essa falta de conscientização. (E-2)

Muitas vezes por negligência da pessoa mesmo não usam, mas o correto é usar (EPI's). (E-7)

Falta de compromisso deste profissional com a vida dos pacientes. (E-1 I)

Um outro aspecto foi apontado pelos profissionais de enfermagem como ação capaz de conscientização e geração de mudanças, foi e a educação em saúde, traduzida como sinônimo de "reciclagem", "orientação", verbalizado nas seguintes expressões:

Esse funcionário precisa fazer uma reciclagem, tudo Que você vai manusear precisa lavar as mãos, se ele não faz isso ele tem Que fazer uma reciclagem com certeza. (E-4)

\section{Mais informações, tais como curso de reciclagem.... (E-9)}

Deve ser feito um trabalho educativo para Que essas pessoas fiquem sabendo a real importância dos EPI's. (E-10)

A Qualificação dos funcionários, reciclagem, cursos de aprendizagem. (E-I I)

A formação dos profissionais com uma percepção de prevenção e uma visão mais ampliada de mundo, talvez seja, na realidade, um dos desafios Que o ensino na área de saúde enfrenta. Pressupõe-se Que o ensino deva favorecer a prática de condutas corretas e oportunizar atividades práticas pertinentes à prevenção e ao controle de infecção Que atendam às necessidades do respectivo exercício profissional.

Sem descartar a relevância Que tem as máQuinas e instrumentos Que auxiliam a produção do trabalho em saúde, na definição de diagnósticos e terapêuticas, ressaltamos neste contexto, a importância/papel do profissional de saúde, identificado anteriormente por Semmelweis, como agente ativo na transmissão 
de IH. Na prática, nota-se Que os profissionais participam de ações educativas, conhecem o tema "infecção hospitalar", sabem da importância de lavar as mãos, e, no entanto, não aderem às técnicas, normas e modificações nos serviços de saúde.

O uso correto de máscara, luvas, óculos e principalmente a lavagem das mãos antes e depois de manipular com o paciente. (E-9)

\section{...lavar as mãos é imprescindível para evitar infecção hospitalar.} $(\mathrm{E}-3)$

O processo educativo está intimamente relacionado às subjetividades individuais de cada profissional, pois se sabe que a prática em saúde, embora embasada em uma teoria científica, é profundamente dependente dos valores morais, éticos, ideológicos e subjetivos destes profissionais, envolvendo interpretação, ajuizamento e decisão pessoal na aplicação do conhecimento científico às situações concretas e singulares. Caracterizando assim as representações sociais de cada indivíduo na prática cotidiana.

\section{CONSIDERAÇÕES FINAIS}

Neste estudo percebeu-se Que os profissionais de enfermagem formularam conceito sobre infecção hospitalar conforme o ambiente em Que estão inseridos, baseados em suas percepções e conhecimentos pré-estabelecidos. A representação elaborada sobre infecção hospitalar foi uma definição associada à contaminação, principalmente através de materiais e equipamentos hospitalares. Valorizando estes meios como determinante das infecções hospitalares, desprezando medidas imprescindíveis na prevenção das infecções tais como: lavagem das mãos e uso de EPI's, apesar de reconhecerem sua importância.

Observou-se que existe conhecimento técnico com fundamentação científica sobre infecção hospitalar, no entanto reconhecem Que não adotam normalmente as medidas de prevenção e controle, expondo-se ao risco.

A estrutura organizacional e funcional aparece como representação social das condições de trabalho, interferindo diretamente nas práticas profissionais, influenciando na Qualidade da assistência e determinando comportamentos. Constituindo assim um determinante da não adesão dos profissionais de enfermagem as técnicas e rotinas instituídas para prevenção e controle das infecções hospitalares.

As medidas de prevenção e controle das infecções hospitalares devem ser um hábito entre os profissionais de saúde, e a adesão à sua prática um desafio a ser atingido. Para Que esses objetivos sejam alcançados, os profissionais deverão ser conscientizados, motivados e orientados em um processo permanente.

Sabemos, portanto, Que hodiernamente existe uma tendência/ corrente influenciando modificações nos cursos de graduação na área da saúde e uma preocupação premente em inserir a temática da prevenção e controle das infecções nas disciplinas curriculares. No entanto em relação aos técnicos e auxiliares de enfermagem, Questionamos-nos sobre os cursos profissionalizantes, as metodologias empregadas, e até sobre a capacitação profissional. Indagamos, o Que realmente vêm sendo feito neste sentido? O Que precisa ser feito?

Estamos convencidos de Que novas estratégias são necessárias para obtenção de maior obediência as rotinas básicas para prevenção e controle das infecções hospitalares, uma vez Que as medidas até então adotadas têm sido de pouco sucesso.

É necessário, então, ressaltar Que o foco de nossas atenções deve ser direcionado aos profissionais de saúde de modo geral, pois eles são os sujeitos ativos deste processo. Portanto, deve-se buscar entender as representações sociais de cada realidade, para assim instituir ações realmente eficazes na prevenção e controle das infecções.

\section{REFERÊNCIAS}

I. Moscovici S. Representações sociais: investigações em psicologia social. Petrópolis (RJ): Vozes; 2003.

2. Nóbrega SM. Sobre a teoria das representações sociais. In: Moreira ASP, Jesuíno IC, organizadores. Representações sociais: teoria e prática. 2 2a. ed. João Pessoa (PB): Universitária; 2003. p. 5 I-80.

3. Arruda A. Teoria das representações sociais e teorias de gênero. Cad Pesquisa 2002; 117:127-47.

4. Jodelet D. As representações sociais. Rio de Janeiro (RJ): UERJ; 2001 .

5. Matheus MCC. Fustinoni SM. Pesquisa Qualitativa em enfermagem. I ${ }^{\text {a. }}$ ed. São Paulo (SP): LMP Editora; 2006.

6. Minayo MCM. Pesquisa social: teoria, método e criatividade. 8. ed. Petrópolis (RJ): Vozes; 1998.

7. Ministério da Saúde (BR). Conselho Nacional de Saúde. Resolução 196/96. Diretrizes e normas regulamentadoras de pesquisa envolvendo seres humanos. Brasília (DF): Ministério da Saúde; 1996.

8. Ministério da Saúde (BR). Portaria no 2.616, de 12 de maio de 1998. Dispõe sobre o programa de controle infecção hospitalar.
Diário oficial da República Federativa do Brasil, Brasília (DF); 1998.

9. Bolick D, Bruner D W, Brady C. Segurança e controle de infecção. Rio de Janeiro (RI): Reichman \& Affonso; 2000.

10. Moreira ASP, Jesuíno, JC. Representações sociais: teoria e prática. $2^{\text {a. }}$ ed. João Pessoa (PB): Universitária; 2003.

1 I. Nasi LA. Rotinas em pronto-socorro. $2^{\text {a. }}$ ed. Porto Alegre (RS): Artmed; 2005.

12. Pereira MS, Prado MA, Leão ALM, Souza DN. Avaliação de serviços de apoio na perspectiva do controle de infecção hospitalar. Rev Eletr Enf 1999; I(1).

13. Triple A FV, Pereira MS, Hayashida M, MoriyaTM, Souza ACS. O ensino do controle de infecção: um ensaio teórico-prático. Rev Latino-am Enfermagem 2003; 1 I (2): 245-50.

14. Catteteblom BL, Lima SL. Lavagem das mãos. In: Enciclopédia da Saúde - Infecção Hospitalar. Rio de Janeiro (R)): Medsi; 2001.

15. Moura MEB, Campelo SMA, Brito FCP, Batista OMA, Araújo TME, Oliveira ADS. Infecção hospitalar: estudo de prevalência em um hospital público de ensino. Rev Bras Enferm 2007; 60 (4): $4 \mid 6-2 I$ 